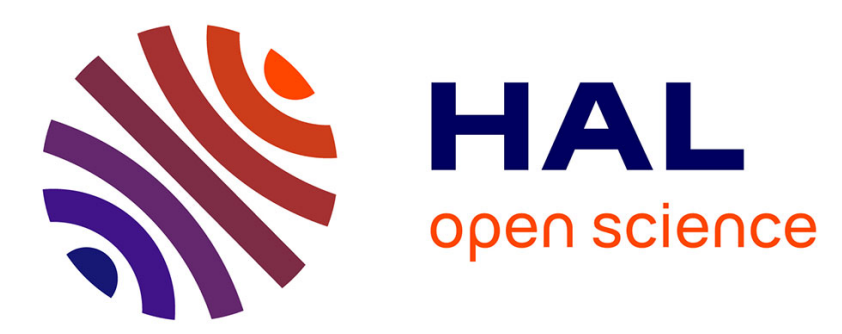

\title{
Obtention d'un plasma de forte densité (5 x 1011cm-3) dans une décharge multipolaire de grande dimension
}

\author{
M. Benhassine, J.C. Bourdessol, C. Gauthereau, J. Godiot, G. Matthieussent,
} Jean-Louis Rauch

\section{- To cite this version:}

M. Benhassine, J.C. Bourdessol, C. Gauthereau, J. Godiot, G. Matthieussent, et al.. Obtention d'un plasma de forte densité ( 5 x 1011cm-3) dans une décharge multipolaire de grande dimension. Revue de Physique Appliquée, 1984, 19 (7), pp.545-553. 10.1051/rphysap:01984001907054500 . jpa-00245224

HAL Id: jpa-00245224

https://hal.science/jpa-00245224

Submitted on 1 Jan 1984

HAL is a multi-disciplinary open access archive for the deposit and dissemination of scientific research documents, whether they are published or not. The documents may come from teaching and research institutions in France or abroad, or from public or private research centers.
L'archive ouverte pluridisciplinaire HAL, est destinée au dépôt et à la diffusion de documents scientifiques de niveau recherche, publiés ou non, émanant des établissements d'enseignement et de recherche français ou étrangers, des laboratoires publics ou privés. 


\title{
Obtention d'un plasma de forte densité $\left(5 \times 10^{11} \mathrm{~cm}^{-3}\right)$ dans une décharge multipolaire de grande dimension
}

\author{
M. Benhassine, J. C. Bourdessol, C. Gauthereau, J. Godiot, G. Matthieussent et J. L. Rauch \\ Laboratoire de Physique des Gaz et des Plasmas (*), et GRECO-I.L.M., \\ Bât. 212, Université de Paris Sud, 91405 Orsay, France
}

(Reçu le 17 janvier 1984, révisé le 26 mars, accepté le 3 avril 1984)

\begin{abstract}
Résumé. - On décrit les caractéristiques et le fonctionnement d'une décharge multipolaire de grande dimension permettant d'obtenir un plasma de densité élevée, présentant ou non un gradient unidimensionnel de densité. Le plasma est produit dans un volume de l'ordre du $\mathrm{m}^{3}$, dans de l'argon $\left(\mathrm{He}, \mathrm{Ar}, \mathrm{N}_{2}\right)$ avec les caractéristiques suivantes : pression entre $10^{-5}$ et $10^{-3}$ torr, densité électronique inférieure à $5 \times 10^{11} \mathrm{~cm}^{-3}$, température électronique comprise entre 3 et $6 \mathrm{eV}$, fluctuation relative de densité de l'ordre de $10^{-3}$.
\end{abstract}

\begin{abstract}
A multipolar discharge has been constructed to produce a high density plasma either uniform or with a one-dimensional density gradient. The main characteristics of the discharge are : volume of the order of $1 \mathrm{~m}^{3}$, pressure of argon $\left(\mathrm{He}, \mathrm{Ar}, \mathrm{N}_{2}\right)$ between $10^{-5}$ torr and $10^{-3}$ torr, electronic density less than $5 \times 10^{11} \mathrm{~cm}^{-3}$, electron temperature between 3 and $6 \mathrm{eV}$, relative density fluctuations of the order of $10^{-3}$.
\end{abstract}

\section{Introduction.}

L'utilisation des décharges électriques où le plasma est produit dans une configuration de champ magnétique multipolaire s'est développée au cours des dix dernières années; en effet ces dispositifs permettent de produire un plasma calme, spatialement uniforme et non magnétisé, dans un grand volume [1]. Le plasma est créé par des électrons rapides (électrons primaires de quelques dizaines de volts) qui ionisent un gaz neutre. Ces électrons sont confinés par des aimants permanents qui tapissent l'enceinte [2,3]; ainsi pour un courant de décharge donné, la densité d'un plasma produit dans un dispositif multipolaire, dépasse de plusieurs ordres de grandeur celle obtenue dans le même dispositif en l'absence d'aimants [1].

De nombreuses expériences de physique et de chimie des plasmas sont réalisées sur ce type de décharge : propagation d'ondes [4], turbulence [5], dépôt et gravure par plasma [6], études de cinétique chimique [7], production d'ions négatifs [8]. Ces décharges multipolaires rendent en effet possible la production de plasmas de gaz atomiques $(\mathrm{H}, \mathrm{He}, \mathrm{Ar}$, $\mathrm{Kr}, \ldots)$ ou moléculaires $\left(\mathrm{N}_{2}, \mathrm{O}_{2}, \mathrm{SiH}_{4}, \mathrm{SiCl}_{4}\right)$ à basse

(*) Laboratoire associé au CNRS, LA 73. pression $\left(10^{-5}\right.$ torr $<p<10^{-3}$ torr $)$ dans de grands volumes (vol. $\sim 1 \mathrm{~m}^{3}$ ), avec des densités maxima de l'ordre de $n \sim 10^{18}$ électrons $\mathrm{m}^{-3}$ et des températures électroniques comprises entre 1 et $10 \mathrm{eV}$. Ces plasmas sont produits en continu, avec des tensions et courants de décharge relativement modérés (vol. $\sim 1 \mathrm{~m}^{3}, p \sim 10^{-4}$ torr, $\mathrm{Ar}, n \approx 10^{18} \mathrm{~m}^{-3}, V \simeq 30 \mathrm{~V}$, $I \simeq 100 \mathrm{~A}$ ).

Un tel dispositif multipolaire fonctionne au Laboratoire de Physique des Gaz et des Plasmas à Orsay. Des études d'ondes et de turbulence y sont en cours, en vue de mieux comprendre une partie de la physique liée aux expériences d'interaction laser matière et aux expériences de modification de l'ionosphère.

Le but de cet article est de présenter les solutions retenues pour ce dispositif multipolaire fonctionnant à densité de plasma élevée, d'analyser le fonctionnement physique d'une telle décharge pour en faire ressortir les limites, et de donner les principaux résultats sur le fonctionnement de la décharge et les caractéristiques du plasma.

\section{Dispositif expérimental.}

L'ensemble du dispositif expérimental (Figs. 1 et 2) est constitué par une enceinte à vide en inox contenant 


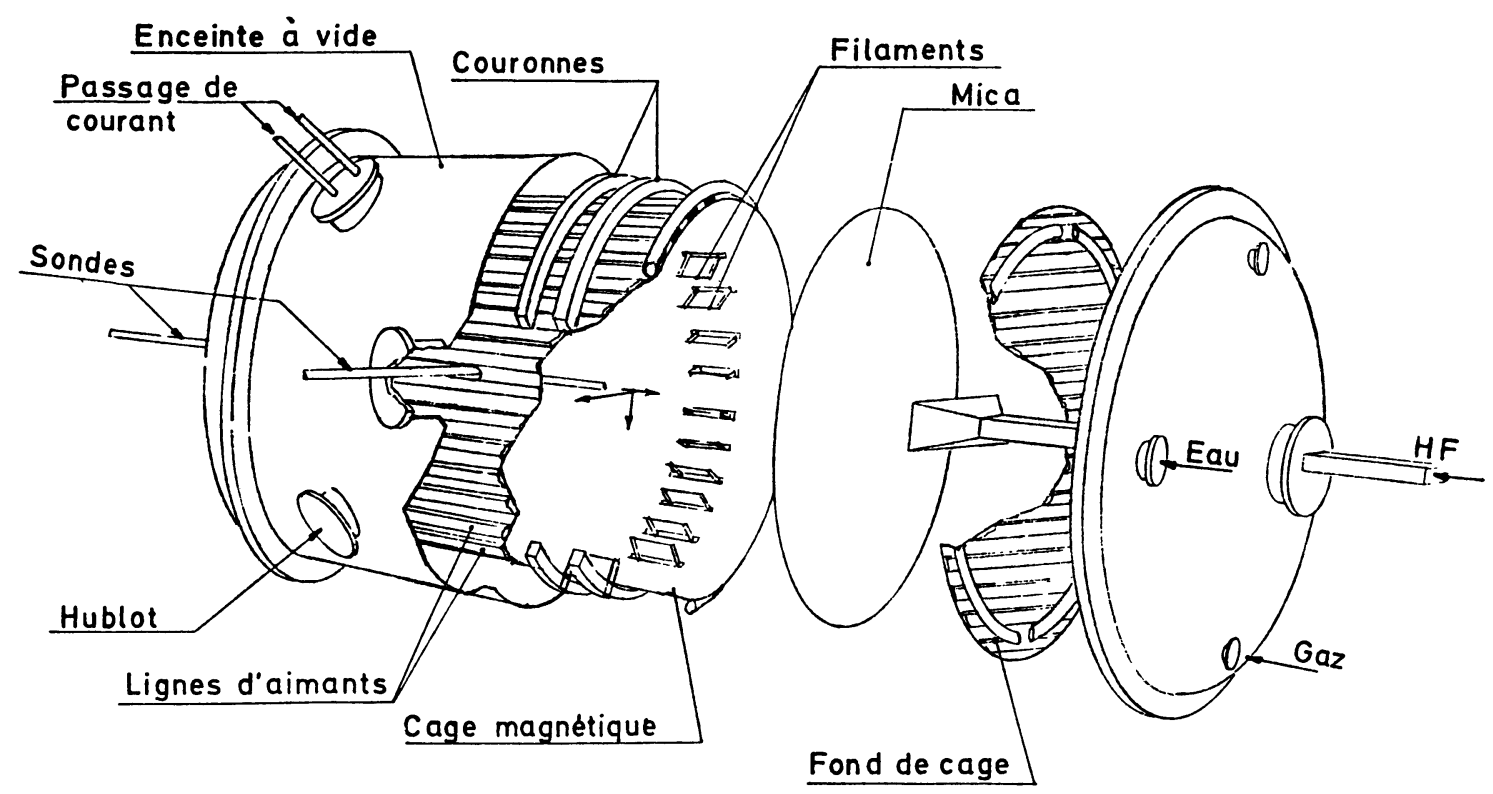

\section{DISPOSITIF MULTIPOLAIRE}

Fig. 1. - Schéma d'ensemble de la décharge multipolaire.

[Schematic view of the multipolar discharge.]

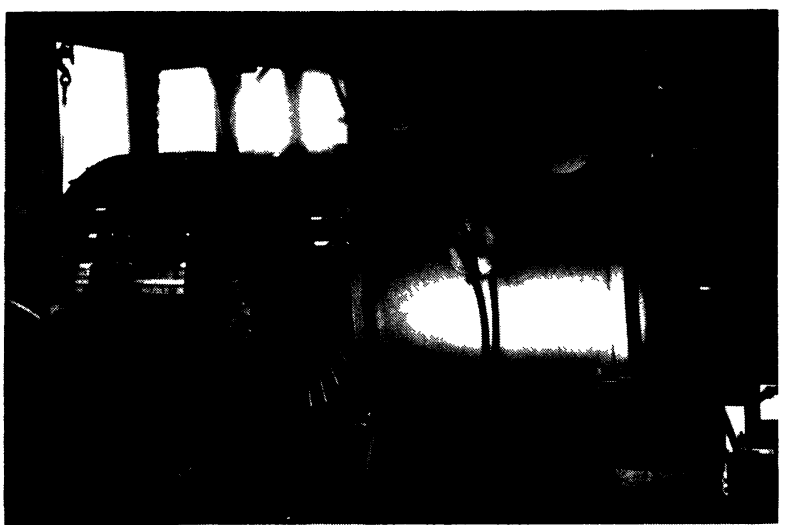

Fig. 2. - Photographie de la décharge multipolaire; cage d'aimants sortie.

[Snapshot of the multipolar discharge.]

une cage cylindrique en cuivre (longueur $1 \mathrm{~m}$, diamètre $1 \mathrm{~m}$ ). Sur les parois extérieures de cette cage sont disposées des rangées d'aimants permanents, de polarité alternée, ainsi que les circuits de refroidissement par circulation d'eau. Sur deux paires de couronnes en cuivre, entourant la cage, sont fixés les supports de filaments. Ceux-ci traversent la cage par des fentes situées le long des génératrices de cette cage. L'isolement électrique entre les supports et la cage est assuré par des tubes d'alumine entourant ces supports. Les filaments de tungstène sont tendus entre les supports ; ils sont chauffés à $2800 \mathrm{~K}$ et émettent des électrons dits primaires. Ceux-ci sont accélérés par une diffé- rence de potentiel de quelques dizaines de volts appliquée entre les filaments et la cage reliée à la masse ; les électrons primaires ionisent alors le gaz entraînant la formation d'un plasma.

2. 1 Champ magnétiQue multipolaire. - Le champ magnétique de surface est obtenu classiquement en utilisant de petits aimants permanents en ferroxdure orienté $330 \mathrm{~K}(L=4 \mathrm{~cm}, e=2 \mathrm{~cm}, h=1 \mathrm{~cm})$, disposées en rangées, de polarité alternée, à l'intérieur de profilés fixés sur la cage et sur les deux flasques d'extrémité. Les rangées sont espacées de six centimètres $(l=6 \mathrm{~cm})$ et forment des circuits magnétiques fermés. Le champ magnétique $\mathbf{B}$ créé par ces aimants est alors donné par [9] :

$$
\mathbf{B}=\frac{2 B_{0} l}{\pi} \nabla \times \lambda(x, y)
$$

et

$$
\lambda(x, y)=\frac{\operatorname{ch} \pi \frac{x}{l} \cos \pi \frac{x}{l}}{\operatorname{ch}^{2} \pi \frac{x}{l}-\cos ^{2} \pi \frac{x}{l}}
$$

où conformément à la figure 3 la direction $\mathrm{O} x$ est perpendiculaire au plan contenant les rangées d'aimants, la direction $\mathrm{O} y$ est dans ce plan et perpendiculaire aux aimants. $\lambda$ est proportionnel au potentiel vecteur et n'a qu'une composante selon $z$. Sur cette figure sont portées les lignes de champ magnétique (qui se confondent avec les lignes à $\lambda$ constant), ainsi que les lignes à module de champ constant. Le 


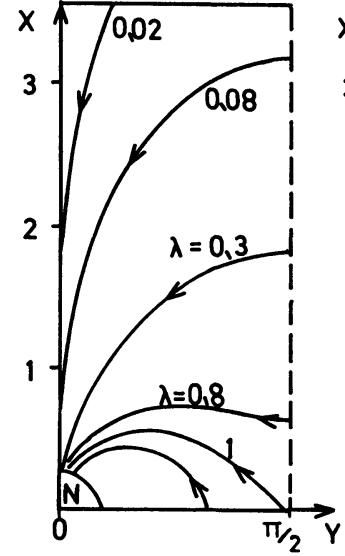

a)

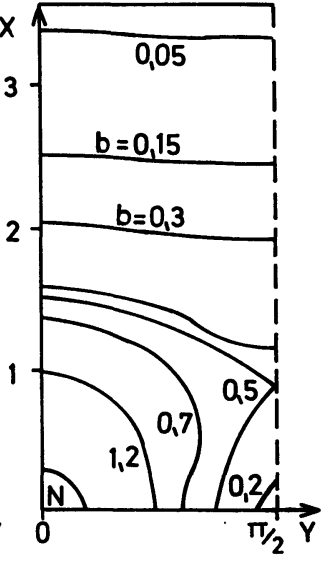

b)
Fig. 3. - Topographie du champ multipolaire $(X=\pi x / l$, $Y=\pi y / l)$; l'aimant est centré en $X=0, Y=0$. a) Lignes de champ, b) lignes à $|\mathbf{B}|$ constant ; les valeurs de $b=B / B_{0}$ sont reportées.

[Multipolar field topography $(X=\pi x / l, \quad Y=\pi y / l)$. A magnet row is centred at $X=0, Y=0$. a) Field lines, b) lines of constant $|\mathbf{B}|$; values of $b=B / B_{0}$ are plotted.]

champ s'annule entre deux aimants $\left(x=0, y=\frac{l}{2}\right)$, et passe par un col le long de la ligne $y=\frac{l}{2}$ en $x=0,9 l / \pi$ où il atteint la valeur $B_{0}$. Les lignes de champ se rejoignent sur les aimants pour y former des cornets. La longueur caractéristique de décroissance du champ magnétique est $\frac{l}{\pi}$.

La figure 4 montre la variation du champ magnétique mesurée à l'aplomb d'une rangée d'aimants et entre ceux-ci. Le champ magnétique est donc localisé

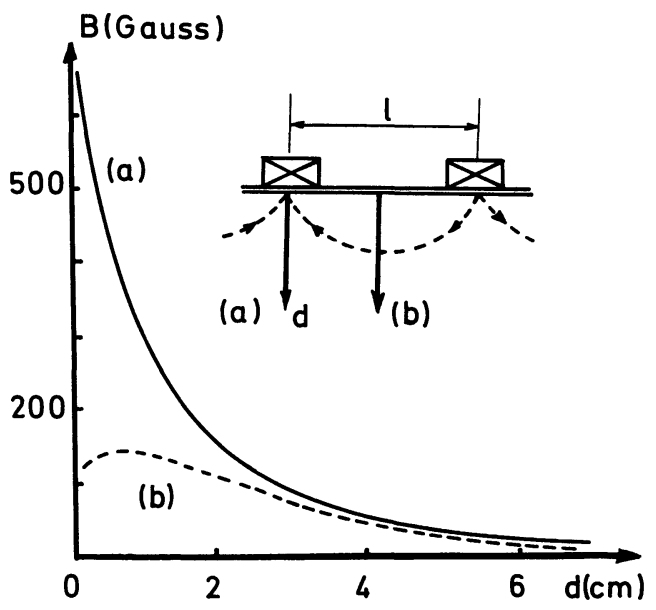

Fig. 4. - Variation du champ magnétique dans l'enceinte en fonction de la distance à la paroi $d$. a) A l'aplomb d'une rangée d'aimants, b) entre deux aimants.

[Variation of the modulus of the magnetic field as a function of the distance to the wall. a) In front of a row of magnets; b) between two rows.] au voisinage de la paroi limitant le plasma, et sur une profondeur d'une dizaine de centimètres.

Le rôle principal de ce champ magnétique de surface est de réfléchir les électrons primaires (énergie de quelques dizaines d'eV) et ainsi d'augmenter leur densité, rendant possible l'entretien d'un plasma dans un dispositif multipolaire jusqu'à des pressions de l'ordre de $10^{-5}$ torr. A cette pression, le libre parcours typique d'un électron primaire, pour des collisions ionisantes, est d'environ une centaine de mètres et le maintien d'une décharge dans un dispositif sans champ magnétique s'avère alors impossible. Quant aux ions et électrons du plasma (températures respectives de l'ordre d'une fraction d'eV et de quelques $\mathrm{eV}$ ), ils diffusent vers les parois sous l'effet conjugué des champs électriques de charge d'espace (quelques volts entre le centre de la décharge et la cage) et du champ magnétique. Ainsi, entre deux dispositifs analogues, fonctionnant à même courant de décharge, l'un avec champ magnétique de surface, l'autre sans, l'augmentation de densité au centre peut atteindre un facteur 100 alors que le temps de vie du plasma reste pratiquement identique, indiquant un mauvais confinement du plasma [10], ce qui a été confirmé théoriquement [9]. Lorsque le libre parcours d'ionisation devient inférieur aux dimensions caractéristiques de l'enceinte, le champ magnétique n'augmente plus la densité des électrons primaires. Il existe donc une pression au-dessus de laquelle les dispositifs multipolaires ne présentent plus d'intérêt.

Par ailleurs, l'analyse du mouvement des électrons primaires dans le champ multipolaire montre que la perte des électrons primaires se fait, au niveau des cornets, par des fentes dont la largeur $\Delta h_{\mathrm{p}}$ est donnée par:

$$
\Delta h_{\mathrm{p}}=\left(\frac{2 \pi b}{l}\right)^{2} \frac{m_{\mathrm{e}} V_{\mathrm{p}}}{e B_{0}} \quad B_{0}=\frac{B_{\mathrm{s}} \pi^{2} a^{2}}{l^{2}}
$$

où $b$ désigne la distance entre l'axe d'une rangée d'aimants supposée cylindrique (rayon $a$ ) et la surface de la cage, $V_{\mathrm{p}}$ la vitesse des primaires, $B_{\mathrm{s}}$ le champ à la surface des aimants. On voit donc que si le nombre de rangées d'aimants est augmenté, la surface totale de fuite des primaires va augmenter. D'autre part, si le champ au col est tel que :

$$
\frac{2 \pi m_{\mathrm{e}} V_{\mathrm{p}}}{e B_{0} l}>1
$$

les électrons primaires vont fuir entre les cornets, par les cols. Il existe donc un optimum pour l'écartement entre les rangées d'aimants qui, expérimentalement, s'est avéré de l'ordre de $5 \mathrm{~cm}$; cet optimum est très peu prononcé. La théorie comme l'expérience montrent que, pour les électrons et les ions, la largeur du trou de fuite $\Delta h$ est proportionnelle au rayon de giration hybride :

$$
\Delta h \simeq \frac{2 l}{\pi}\left(\frac{r_{\mathrm{e}} r_{\mathrm{i}}}{\lambda_{\mathrm{e}} \lambda_{\mathrm{i}}}\right)^{1 / 2}
$$


où $r$ et $\lambda$ désignent respectivement les rayons de giration et les libres parcours moyens des électrons et des ions (indices e et i).

Le champ multipolaire de surface agit donc sur les primaires en les réfléchissant et en les empêchant d'être perdus à la paroi; ce champ, comme cela sera discuté dans le paragraphe consacré aux caractéristiques du plasma, capture aussi les électrons primaires et assure l'uniformité d'un tel plasma.

\subsection{ENCEINTE ET POMPAGE. - L'enceinte est prévidée} par une pompe primaire de $100 \mathrm{~m}^{3} \mathrm{~h}^{-1}$; puis le pompage est assuré par une pompe à diffusion de $3500 \mathrm{ls}^{-1}$. Le vide limite est de l'ordre de $10^{-6}$ torr. Le gaz ( $\mathrm{Ar}, \mathrm{He}, \mathrm{Ne})$ est introduit en continu; les pressions habituelles de fonctionnement sont comprises entre quelque $10^{-5}$ torr et quelque $10^{-3}$ torr. Cette enceinte est munie de six hublots et de passages permettant des diagnostics optiques et l'introduction de diverses sondes.

2.3 Production du Plasma (Fig. 5). - Les électrons primaires, responsables de l'ionisation du gaz neutre, sont émis par 80 filaments de tungstène de $3 / 10 \mathrm{~mm}$ de diamètre et chacun de $10 \mathrm{~cm}$ de longueur; ces filaments, répartis en deux couronnes sont situés à $10 \mathrm{~cm}$ de la cage en cuivre, dans une région où le champ magnétique peut être considéré comme nul.

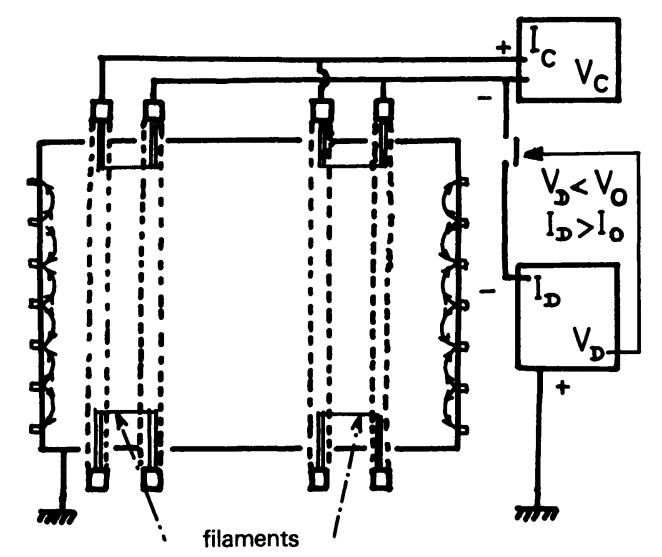

Fig. 5. - Alimentation électrique de la décharge et sécurité.

[Electrical set up.]

Une alimentation, régulée en tension (750 A, $15 \mathrm{~V})$, les chauffe en parallèle à environ $2800 \mathrm{~K}$; ainsi la puissance dissipée par filament ne varie pas en cas de rupture de certains d'entre eux, et lors de l'amorçage de la décharge, la puissance de chauffage reste sensiblement constante, la présence du courant de décharge entraînant une baisse du courant de l'alimentation de chauffage.

Pour une température donnée, ces filaments peuvent émettre un courant électronique maximum, $I_{\mathrm{s}}$, donné par la loi de Richardson-Dushman [11]. La décharge est initiée en portant les filaments à un potentiel de - $60 \mathrm{~V}$ par rapport à la cage en cuivre, à l'aide d'une alimentation régulée en courant. Une fois la décharge établie, la tension baisse et le courant est régulé à une valeur de consigne (quelques dizaines de volts et centaines d'ampères). Par ailleurs, la tension est principalement appliquée au voisinage immédiat du filament, dans une gaine dont l'épaisseur est de l'ordre d'une dizaine de longueurs de Debye.

Si un arc se forme, le courant restant constant, la tension baisse; un système de sécurité coupe alors l'alimentation en quelques microsecondes en bloquant les transistors assurant la régulation (Fig. 5).

\subsection{TyPES DE FONCTIONNEMENT ET DIAGNOSTICS. -} Le dispositif peut fonctionner soit en configuration multipolaire complète (le plasma est alors homogène), soit en présence d'une paroi en mica disposée perpendiculairement à l'axe du cylindre. Cette feuille de mica limite spatialement le champ magnétique de surface que voient les électrons primaires. La population des électrons primaires et l'ionisation au voisinage de la feuille sont modifiées; un gradient longitudinal de densité est ainsi créé. Cette feuille fournit aussi une bonne condition aux limites pour le plasma et pour des ondes électromagnétiques injectées à l'aide d'un cornet hyperfréquence (Fig. 1).

A une extrémité de l'enceinte, un passage permet l'introduction d'un dispositif d'excitation d'ondes (cornet hyperfréquence, canon à électrons). A l'autre extrémité de l'enceinte, est disposée (Fig. 6) une table $(z, r, h)$ à trois degrés de libertés, qui, à l'aide de moteurs, déplace une sonde à l'intérieur de l'enceinte dans un volume défini par $(z=1 \mathrm{~m}, r=h=0,3 \mathrm{~m})$. Par rapport à la rotule $\mathrm{R}$, l'extrémité $\mathrm{S}$ de la sonde reste symétrique du point $M$ situé à l'intersection de l'axe vertical de la table avec l'axe horizontal $\mathbf{M}^{\prime} \mathbf{M}^{\prime \prime}$.

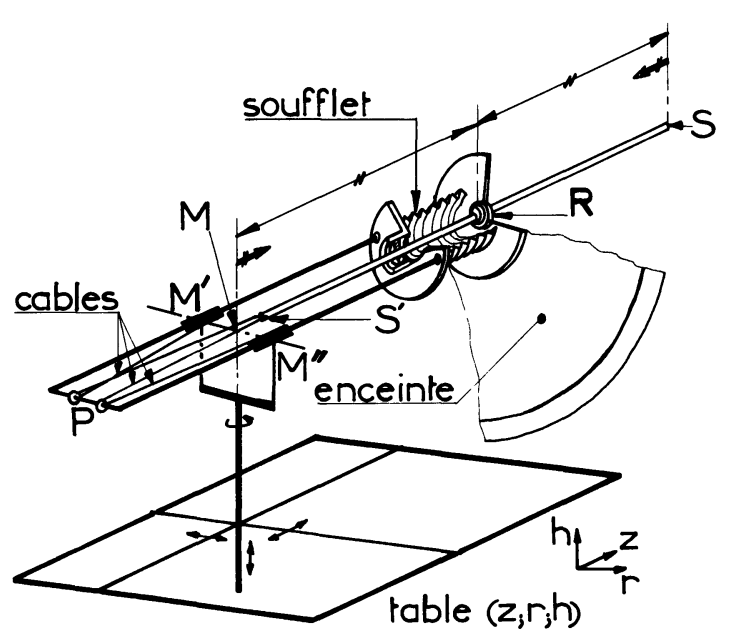

Fig. 6. - Schéma de principe de la sonde mobile. Le point $M$ entraîné par les moteurs reste symétrique de l'extrémité de la sonde, par rapport à la rotule grâce à l'utilisation du câble de rappel.

[Schematic design of the movable probe.] 
En $\mathbf{M}^{\prime}$ et $\mathbf{M}^{\prime \prime}$, situés aux extrémités de la fourchette entraînée par la table, sont centrées deux glissières. Dans celles-ci coulissent deux tiges rigides de longueur constante reliées, d'un côté à l'enceinte, de l'autre à un axe comportant deux poulies $P$. L'extrémité $S^{\prime}$ de la sonde est reliée aux points $\mathbf{M}^{\prime}$ et $\mathbf{M}^{\prime \prime}$ à l'aide de deux câbles passant par les poulies $\mathrm{P}$. La longueur de ces câbles est ajustée une fois pour toutes pour réaliser la symétrie M, R, S. La mise en tension des câbles est assurée par la différence de pression entre l'enceinte et l'extérieur.

Le point $M$ étant déplacé par la table vers l'avant, la sonde recule de la même longueur et la symétrie se conserve; ainsi si $\mathbf{M}$ décrit une courbe, le point $\mathbf{S}$ décrira la courbe symétrique par rapport à la rotule.

L'étanchéité du passage de la sonde dans l'enceinte est réalisée à l'aide d'un tombac. Cette sonde est équipée pour pouvoir mesurer simultanément les caractéristiques du plasma (température et densité à l'aide d'une sonde de Langmuir) et des ondes (antenne dipolaire et sonde électrostatique).

2.5 Limitations. - Avant de décrire les caractéristiques des plasmas obtenus, un bref aperçu des problèmes technologiques rencontrés sera donné.

La puissance dissipée sous vide a rendu obligatoire le refroidissement de la cage et, dans le futur des couronnes support de filaments.

La densité maximum de plasma que l'on peut atteindre est limitée par la puissance de chauffage disponible. En effet, le courant émis par les filaments se sature à une valeur voisine de $I_{s}$, très inférieure au courant de décharge disponible (500 A). Pour augmenter le courant de décharge, donc la densité, lorsque $I_{\text {s }}$ est atteint, il faut augmenter fortement la tension de décharge. Les ions sont alors accélérés et bombardent les filaments et les parties non isolées des supports... entraînant la fusion de ceux-ci et la création de plasma métallique.

Pour résumer, dans les conditions actuelles, le dispositif d'Orsay est caractérisé par, $I_{\mathrm{c}}=750 \mathrm{~A}-80$ filaments de tungstène chauffés à une température calculée [11] $T=2800 \mathrm{~K}-V_{\mathrm{c}}=11 \mathrm{~V}$ - longueur et diamètre des filaments $l=10 \mathrm{~cm}, \phi=0,3 \mathrm{~mm}$. Le calcul permet de prévoir une émission thermoionique [11] maximum de $250 \mathrm{~A}$ et une durée de vie (en l'absence de bombardement ionique) d'une trentaine d'heures. On mesure alors, pour un plasma d'argon à $5 \times 10^{-4}$ torr et pour le courant et la tension de chauffage définie précédemment, un courant de décharge maximum de $250 \mathrm{~A}$ pour une tension de $30 \mathrm{~V}$. La densité électronique est alors voisine de $5 \times 10^{11} \mathrm{~cm}^{-3}$, et la durée de vie des filaments de dix heures.

Il est donc envisageable, tout en travaillant avec les mêmes filaments portés à la même température (durée de vie identique), d'en augmenter le nombre en accroissant proportionnellement le courant de chauffage. L'augmentation de la densité serait alors sensiblement proportionnelle à celle du courant de chauffage.

\section{Caractéristiques de la décharge et du plasma.}

Ce dispositif multipolaire a fonctionné avec de l'argon, de l'hélium et de l'azote (potentiels respectifs d'ionisation : 15,$7 ; 24,6 ; 15,6 \mathrm{~V})$. Il a permis d'obtenir des densités voisines de $10^{12}$ électrons $/ \mathrm{cm}^{-3}$ dans l'argon et de $10^{11} \mathrm{~cm}^{-3}$ dans les deux autres gaz. Dans la suite de cet article, seuls les résultats concernant les décharges dans l'argon seront exposés, ainsi que la manière d'obtenir un gradient réglable de densité électronique.

3.1 CaractéristiQues DE LA DÉCHARGe. - Les caractéristiques de décharge (courbe $V=f(I))$ diffèrent fortement selon que l'alimentation de chauffage est régálée en courant ou en tension (Fig. 7). Dans le premier cas, le courant de décharge s'ajoute au courant de chauffage (dans le second, il se retranche) et au fur et à mesure que le courant de décharge croît, l'émission électronique augmente; la saturation du courant de décharge n'apparaît pas, il y a surchauffe des filaments qui risquent de se rompre en chaîne. Pour une alimentation de chauffage régulée en tension, l'effet est inverse, il y a diminution de la puissance de chauffage et apparition d'un courant de décharge limite au-delà duquel la tension de décharge se met à croître ainsi que la densité; le bombardement ionique augmente alors.

La figure 8 montre à différentes pressions et pour différents courants de chauffage les caractéristiques

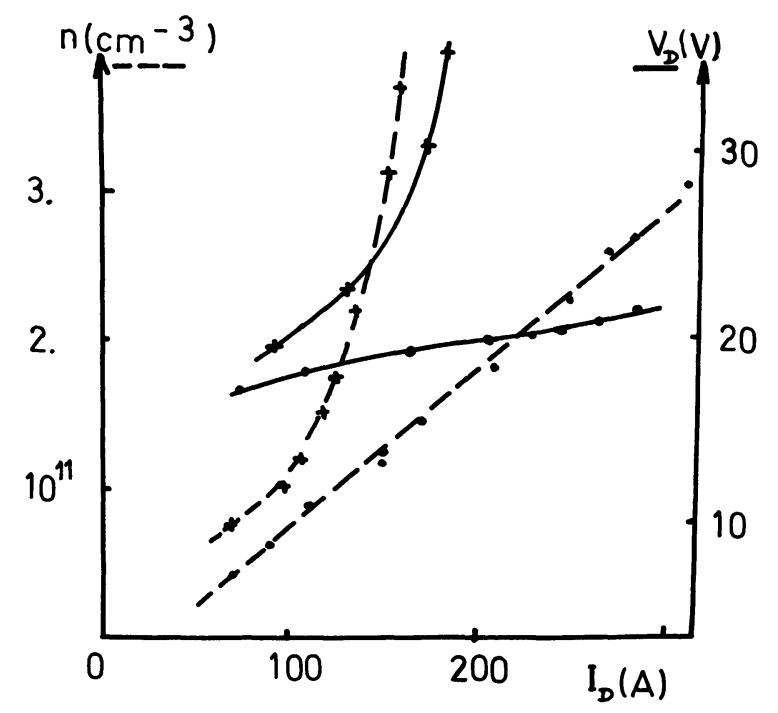

Fig. 7. - Variation de la tension de décharge $V_{\mathrm{D}}(-)$ et de la densité (- - -) au centre $n_{0}$ en fonction du courant de décharge $(\odot)$ Alimentation de chauffage régulée en courant $\left(p=5 \times 10^{-4}\right.$ torr, $\left.I_{c}=700 \mathrm{~A}\right) ;(+)$ alimentation de chauffage régulée en tension $\left(p=10^{-3}\right.$ torr $\left.I_{c}=650 \mathrm{~A}\right)$.

[Discharge voltage $V_{\mathrm{D}}(-)$ and density at the centre, $n_{0}$, as a function of the discharge current, $I_{\mathrm{c}}(---)$. (๑) Heating power supply is in current mode $\left(p=5 \times 10^{-4}\right.$ torr, $\left.I_{\mathrm{c}}=700 \mathrm{~A}\right) ;(+)$ heating power supply is in voltage mode $\left(p=10^{-3}\right.$ torr, $\left.I_{\mathrm{c}}=650 \mathrm{~A}\right)$.] 


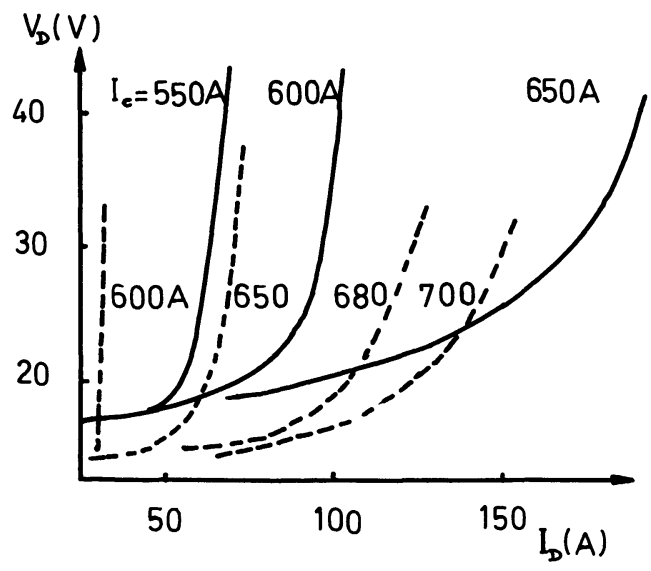

Fig. 8. - Caractéristique de décharge pour différents courants de chauffage $I_{\mathrm{c}}$ à deux pressions différentes : (- -) $p=1,2 \times 10^{-3}$ torr; (-) $p=5 \times 10^{-4}$ torr.

[Voltage current characteristic of the multipolar discharge with heating current as parameter for two different pressions. $(--) p=1,2 \times 10^{-3}$ torr ; (-) $p=5 \times$ $10^{-4}$ torr.]

de décharges obtenus à tension de chauffage constante. A courant de chauffage et de décharge identique, la tension de décharge croît lorsque la pression diminue.

3.2 CaractéristiQues du plasma. - La densité et la température du plasma ont été mesurées à l'aide d'une sonde plane de Langmuir [12]. La figure 9 donne la variation spatiale de la densité électronique (le long du rayon $r$, et dans l'axe, le long de $z$ ), mesurée (croix) et déduite d'un modèle théorique [13]. Ce

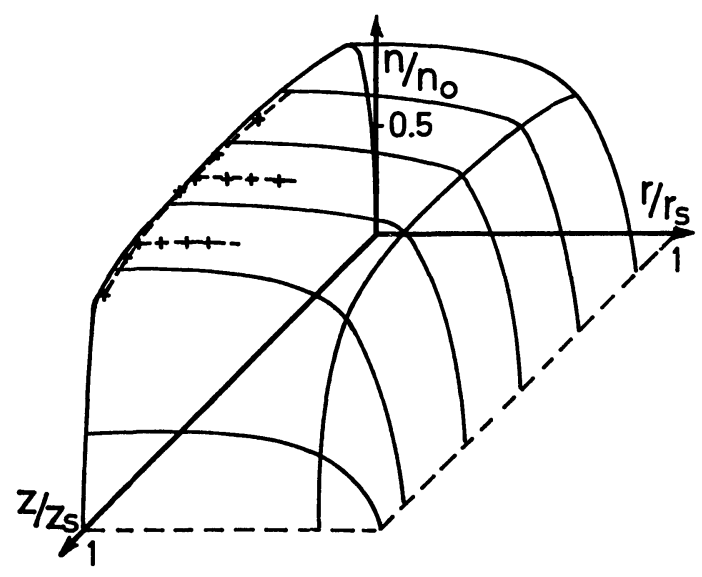

Fig. 9. - Variation spatiale de la densité du plasma, $r$ est selon le rayon, $z$ selon l'axe de la décharge $\left(r_{s}, z_{\mathrm{s}}\right.$ position des parois). Les croix et pointillés sont expérimentaux. La ligne pleine provient de la théorie. Conditions expérimentales $p=10^{-3}$ torr, $I_{\mathrm{D}}=150 \mathrm{~A}, V_{\mathrm{D}}=25 \mathrm{~V}, n_{0}=2 \times$ $10^{11} \mathrm{~cm}^{-3}$.

[Spatial variation of the plasma density $\left(r_{\mathrm{s}}, z_{\mathrm{s}}\right.$ are the position of the walls). Dots and shaded lines experiments; full line theory. The experiments is performed with $p=10^{-3}$ torr, $I_{\mathrm{D}}=150 \mathrm{~A}, V_{\mathrm{D}}=25 \mathrm{~V}$ and $n_{0}=2 \times 10^{11} \mathrm{~cm}^{-3}$.] modèle repose sur le fait, constaté expérimentalement, que le champ magnétique ne joue que très faiblement sur le confinement du plasma [10]. Ce champ piège les électrons primaires au voisinage de la paroi, entraînant, localement, une augmentation de l'ionisation du gaz : la très grande uniformité spatiale de ces plasmas en résulte. En effet, l'ionisation ayant lieu en majeure partie au voisinage de la paroi, très peu d'électrons et d'ions sont créés dans le volume de la décharge où le champ magnétique est nul; le gradient de densité, nécessaire à l'évacuation des particules créées, est alors localisé au voisinage de la paroi. La stabilité de tels plasmas (taux de fluctuation de la densité $\left.\frac{\delta n}{n} \sim 10^{-3}\right)$ résulte de la faible densité de courant en tout point de la décharge.

La figure 10 donne la variation longitudinale de la densité électronique (ramenée à la densité au centre $n_{0}$ ) pour différentes pressions d'argon. Lorsque celle-ci est supérieure à quelque $10^{-3}$ torr, le libre parcours moyen des électrons primaires devient plus petit que la dimension de l'enceinte, l'ionisation devient uniforme dans le volume de la décharge, et le profil de densité devient parabolique [14].

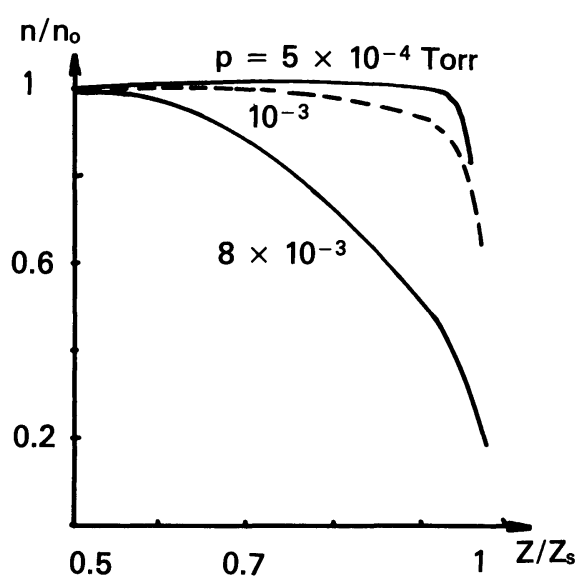

Fig. 10. - Variation axiale de la densité électronique pour différentes pressions $p$.

[Axial variation of the plasma density for different pressures $p$.]

Sur la figure 11 sont reportées, pour deux pressions différentes, les caractéristiques $\left(V_{\mathrm{D}}, I_{\mathrm{D}}\right)$ ainsi que la variation de la densité au centre du dispositif en fonction du courant de décharge. Entre $10^{-4}$ et $10^{-3}$ torr, le potentiel de plasma, $V_{\mathrm{p}}$, est compris entre $-2 \mathrm{~V}$ et quelques $\mathrm{V}$. A une pression donnée, la température électronique est indépendante du courant de décharge tant que la tension de décharge reste sensiblement constante, elle croît ensuite avec celle-ci.

La figure 12 représente à courant de décharge et tension de chauffage constants, les variations de la densité et de la température électronique au centre 


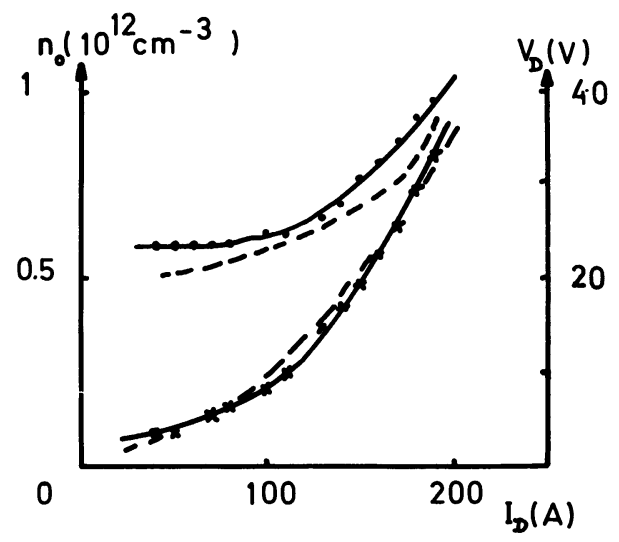

Fig. 11. - Densité $(x)$ et tension de décharge $(\bullet)$ en fonction du courant de décharge pour deux pressions, le courant de chauffage $I_{\mathrm{c}}=650 \mathrm{~A}$. ( $\left.\longrightarrow\right) p=5 \times 10^{-4}$ torr; $(--)^{-}$ $p=10^{-3}$ torr.

[Plasma density $(x)$ and discharge voltage $(\odot)$ as a function of the discharge current, the heating current is $I_{c}=650 \mathrm{~A}$. $(\longrightarrow) p=5 \times 10^{-4}$ torr ; (- - $) p=10^{-3}$ torr.]

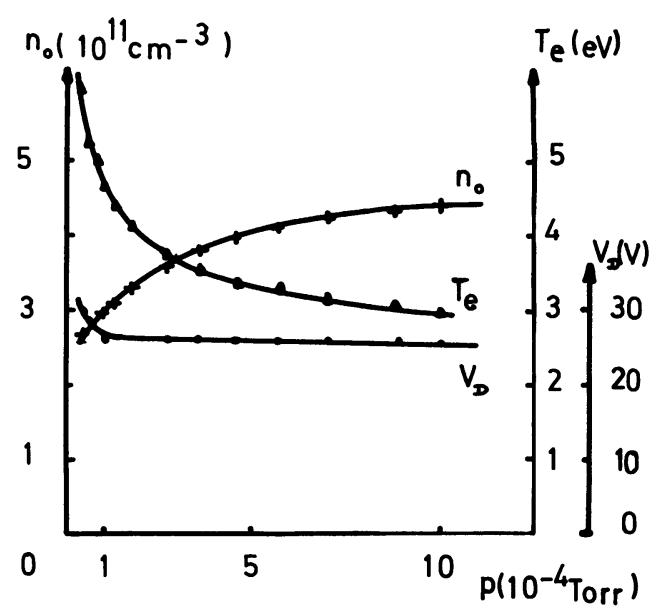

Fig. 12. - Váriation de la densité $(+)$, de la tension de décharge $(\odot)$ et de la température $(\Delta)$ en fonction de la pression d'argon $p$ pour un courant de décharge fixé $(150 \mathrm{~A})$ et de chauffage $(700 \mathrm{~A})$.

[Density $(x)$, discharge voltage $(\odot)$ and temperature $(\Delta)$ as a function of argon pressure for a fixed heating current of $700 \mathrm{~A}$ and discharge current of $150 \mathrm{~A}$.]

de la décharge, ainsi que les variations du potentiel de plasma, en fonction de la pression $p$ entre $5 \times 10^{-5}$ torr et $10^{-3}$ torr. Dans cette gamme de pression, la température varie comme $p^{-0,2}$ sans qu'une justification théorique ait été trouvée.

En résumé, nous avons obtenu, dans ce dispositif, à des pressions comprises entre quelque $10^{-5}$ torr et $10^{-3}$ torr, un plasma d'argon, calme et homogène, dont la densité est de l'ordre de $5 \times 10^{11} \mathrm{~cm}^{-3}$ (degré d'ionisation de l'orḑre de quelques pour cent) avec des températures électroniques comprises entre 3 et $6 \mathrm{eV}$. Les fréquences et longueurs caractéristiques de ce plasma sont données dans l'appendice A. Il faut remarquer que ce plasma peut être considéré comme complètement ionisé : les fréquences de collisions coulombiennes des électrons sur les ions sont d'un ordre de grandeur supérieur à celle des électrons sur les neutres.

3.3 CRÉATION D'UN GRADIENT LONGITUDINAL DE DENSITÉ. - Pour établir un gradient de densité, une feuille de mica est disposée transversalement à l'axe du cylindre; la distance de cette feuille à la paroi magnétisée de la cage est ajustable $(d=10 \mathrm{~cm}$ au maximum). Son rôle est de limiter spatialement le champ magnétique de surface vu par les électrons primaires et donc de modifier l'ionisation [13]. Ainsi, lorsque la feuille de mica est à une distance de $10 \mathrm{~cm}$ du fond de l'enceinte, le champ magnétique de surface est nul, les électrons primaires ne sont plus piégés au voisinage de cette feuille et la répartition spatiale de la densité électronique est donnée par la figure 13 (croix et tirets). Les courbes en trait plein ont été calculées avec le modèle exposé précédemment où l'on a supprimé l'augmentation de l'ionisation au voisinage de la feuille de mica.

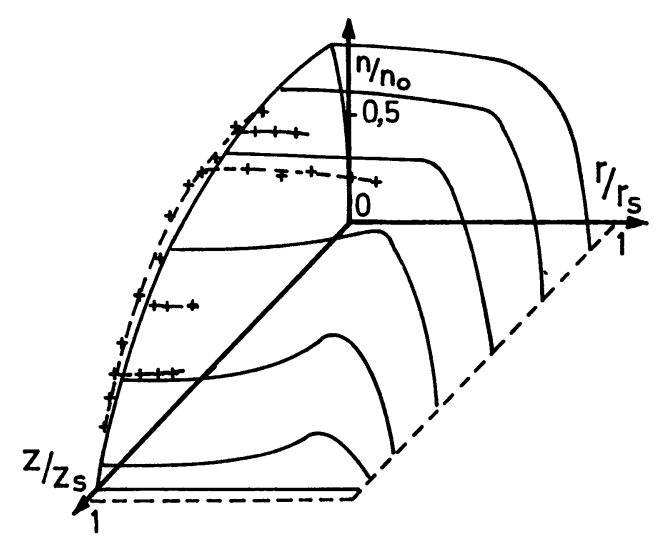

Fig. 13. - Variation spatiale de la densité électronique, en présence d'une feuille de mica disposée perpendiculairement à l'axe du caisson et à $10 \mathrm{~cm}$ du fond. Les croix et pointillés sont expérimentaux. Les courbes en traits pleins ont été calculées.

[With the mica sheet disposed transverse to the axis of the discharge and at $10 \mathrm{~cm}$ from the wall, spatial variation of the plasma density. Same conditions as in figure 9.]

Les conditions expérimentales sont les suivantes : pression d'argon de $10^{-3}$ torr, courant et tension de décharge $150 \mathrm{~A}$ et $25 \mathrm{~V}$, densité maximum de $2 \times 10^{11} \mathrm{~cm}^{-3}$ et température électronique de $3 \mathrm{eV}$. La présence de la feuille de mica crée donc un gradient axial alors que l'uniformité radiale du plasma subsiste.

Le déplacement de la feuille de mica dans le champ magnétique de surface entraîne une variation du 


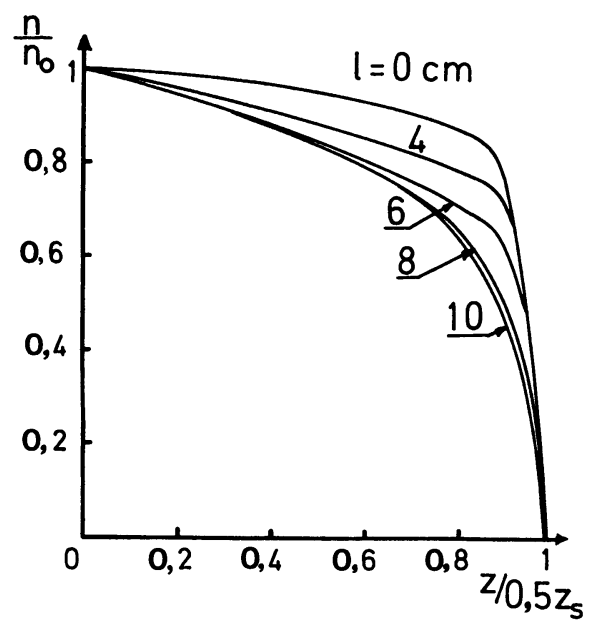

Fig. 14. - Variation axiale de la densité du plasma pour différentes distances $l$ entre la feuille de mica et la paroi. Conditions expérimentales : $n_{0} \simeq 10^{11} \mathrm{~cm}^{-3}, T_{\mathrm{e}}=4 \mathrm{eV}$, $p_{0}=5 \times 10^{-4}$ torr.

[Axial variation of the density for different distances, $l$, between the mica sheet and the wall.]

profil de densité axial comme le montre la figure 14 La longueur caractéristique de gradient

$$
L=\left(\frac{\nabla n}{n}\right)^{-1}
$$

peut donc être ajustée entre $0,3 \mathrm{~m}$ et $3 \mathrm{~m}$.

Par ailleurs, outre la variation axiale de la densité, la présence de la feuille de mica entraîne une variation du potentiel plasma $V_{\mathrm{p}}$ qui est relié à la densité par la loi de Boltzman

$$
n=n_{0} \exp \left(-\frac{V_{\mathrm{p}}}{T_{\mathrm{e}}}\right) .
$$

Le champ électrique est alors de quelques volts par mètre et est donc dirigé (sauf dans la gaine magnétique) le long de l'axe de la décharge. Ce champ électrique, $E$, induit, en présence du champ magnétique de surface, une vitesse de dérive macroscopique du plasma dans la gaine magnétique, égale à $\frac{\mathbf{E} \times \mathbf{B}}{B^{2}}$ et dirigée, selon

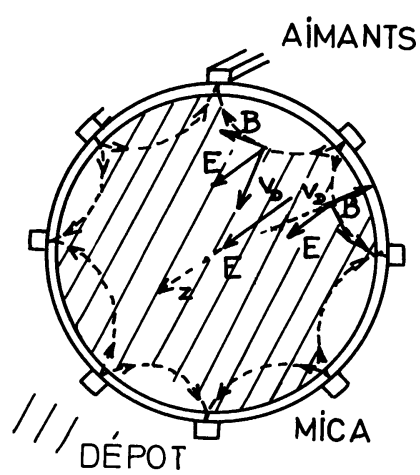

Fig. 15. - Effet sur la feuille de mica de la dérive du plasma en $\mathbf{E} \times \mathbf{B}$.

[Schematic view of the mica sheet showing the effects of the $\mathbf{E} \times \mathbf{B}$ drift on the plasma density.]

le sens de $B$, soit vers l'intérieur, soit vers l'extérieur du plasma avec une périodicité spatiale double de celle des rangées d'aimants comme le montre la figure 15 . La présence ou l'absence de plasma, au voisinage de la paroi est corrélée à celle du dépôt métallique de tungstène sur la feuille de mica.

\section{Conclusion.}

Le plasma obtenu dans le dispositif du Laboratoire de Physique des Gaz et des Plasmas d'Orsay présente des caractéristiques particulièrement intéressantes tant pour la chimie des plasmas que pour des études d'ondes et de turbulence du fait de sa densité élevée obtenue dans un grand volume, d'un taux de fluctuations de densité particulièrement faible et des diagnostics mis en œuvre. En particulier, l'obtention d'un gradient de densité réglable, permet d'injecter dans un plasma présentant un gradient de densité quasiment unidimensionnel, des ondes électromagnétiques et de les faire se propager jusqu'à la résonance de plasma (fréquence de l'onde identique à la fréquence locale du plasma). Il est alors possible de simuler, sur un tel dispositif, une partie de la physique liée à l'interaction laser-matière, et aux expériences de modification de l'ionosphère à l'aide d'ondes de haute fréquence.

\section{Appendice A. - PARAMÈTres De la déCharge MUlti POLAIRE.}

Nature du gaz

Pression résiduelle

Densité des atomes neutres

Densité électronique du plasma :

taux d'ionisation

fréquence plasma électronique

fréquence plasma ionique

Température électronique

Températion ionique estimée

$$
\begin{aligned}
& \text { argon } \\
& P_{0}=10^{-4} \text { torr } \\
& n_{0}=3,5 \times 10^{12} \mathrm{~cm}^{-3} \\
& n_{\mathrm{e}}=10^{11} \mathrm{~cm}^{-3} \\
& \beta \simeq 3 \% \\
& v_{\mathrm{Pe}}=3 \times 10^{9} \mathrm{~s}^{-1} \\
& v_{\mathrm{Pi}} \simeq 10^{7} \mathrm{~s}^{-1} \\
& T_{\mathrm{e}}=3 \mathrm{eV} \\
& T_{\mathrm{i}} \simeq 0,2 \mathrm{eV}
\end{aligned}
$$


Energie des électrons primaires

vitesse thermique électronique

vitesse thermique ionique

vitesse acoustique ionique

vitesse des primaires

longueur de Debye électronique

$$
\begin{aligned}
E_{\mathrm{c}} & =50 \mathrm{eV} \\
v_{\mathrm{te}} & =\sqrt{\frac{8}{\pi} \frac{K T_{\mathrm{e}}}{m_{\mathrm{e}}}}=1,2 \times 10^{6} \mathrm{~m} / \mathrm{s} \\
v_{\mathrm{ti}} & =680 \mathrm{~m} / \mathrm{s} \\
C_{\mathrm{s}} & =\sqrt{\frac{K T_{\mathrm{e}}}{m_{\mathrm{e}}}}=3000 \mathrm{~m} / \mathrm{s} \\
v_{0} & =\sqrt{\frac{2 E_{\mathrm{c}}}{m_{\mathrm{e}}}}=4 \times 10^{6} \mathrm{~m} / \mathrm{s} \\
\lambda_{\mathrm{D}} & =\left\{\frac{\varepsilon_{0} K T_{\mathrm{e}}}{n_{\mathrm{e}} e^{2}}\right\}^{1 / 2}=4 \times 10^{-5} \mathrm{~m}
\end{aligned}
$$

Collisions sur les neutres : transfert de quantité de mouvement :

- électrons primaires :

- fréquence des collisions

- électrons du plasma :

$$
\text { - fréquence des collisions }\left(T_{\mathrm{e}}=1 \mathrm{eV}\right)
$$

Collisions ion-neutre avec transfert de charge :

- fréquence des collisions

Collisions ion-neutre sans transfert de charge :

- fréquence des collisions

Collisions ionisantes des primaires sur les neutres :

- fréquence des collisions ionisantes

Collisions Coulombiennes

- collisions électron-ion

- collisions électron-électron

- collisions ion-ion

- collisions primaire-ion

Grandeurs liées au champ magnétique : écartement des aimants valeur du champ à $4 \mathrm{~cm}$ d'un aimant valeur du champ à $8 \mathrm{~cm}$ d'un aimant pulsation de Larmor électronique pulsation de Larmor ionique rayon de Larmor d'un électron du plasma rayon de Larmor d'un électron primaire rayon de Larmor ionique

$$
\begin{aligned}
& v_{50}^{\mathrm{Po}}=1,3 \times 10^{6} \mathrm{~s}^{-1} \\
& v_{1}^{\mathrm{eo}}=5,7 \times 10^{4} \mathrm{~s}^{-1} \\
& v_{\mathrm{io}}^{*}=1,6 \times 10^{3} \mathrm{~s}^{-1} \\
& v_{\text {io }}=2 \times 10^{3} \mathrm{~s}^{-1} \\
& v_{50}^{\mathrm{i}}=3,7 \times 10^{5} \mathrm{~s}^{-\mathrm{v}_{1}} \\
& v_{\mathrm{ei}}=3 \times 10^{-5} n_{\mathrm{i}}\left(T_{\mathrm{e}}^{\mathrm{eV}}\right)^{-3 / 2}=5,8 \times 10^{5} \mathrm{~s}^{-1} \\
& v_{\mathrm{ee}}=5,8 \times 10^{5} \mathrm{~s}^{-1} \\
& v_{\mathrm{ii}}=v_{\mathrm{ee}}\left(\frac{m_{\mathrm{e}}}{M}\right)^{1 / 2}\left(\frac{T_{\mathrm{e}}}{T_{\mathrm{i}}}\right)^{3 / 2}=1,2 \times 10^{5} \mathrm{~s}^{-1} \\
& v_{\mathrm{pi}} \simeq 10^{4} \mathrm{~s}^{-1} \\
& l=6 \mathrm{~cm} \\
& B \simeq 10^{-2} \mathrm{~T}=100 \mathrm{G} \\
& B \simeq 4 \mathrm{G} \\
& \omega_{\text {be }}=1,7 \times 10^{9} \mathrm{rd} / \mathrm{s} \\
& \Omega^{\mathrm{i}}=2,4 \times 10^{4} \mathrm{rd} / \mathrm{s} \\
& \left.\begin{array}{l}
r_{\mathrm{L}}^{\mathrm{e}}=6 \times 10^{-4} \mathrm{~m} \\
r_{\mathrm{L}}^{\mathrm{P}}=2,4 \times 10^{-3} \mathrm{~m}
\end{array}\right\} B=100 \mathrm{G} \\
& r_{\mathrm{L}}^{\mathrm{i}}=0,04 \mathrm{~m}
\end{aligned}
$$

\section{Bibliographie}

[1] Limpaecher, R., Mac Kenzie, K. R., Rev. Sci. Instrum. 44 (1973) 6.

[2] Leung, K. N., Samec, T. K., Lamm, A., Phys. Lett. A 51 (1975) 490.

[3] Leung, K. N., Hershnowitz, N., Romesser, T., Phys. Lett. A 53 (1975) 264.

[4] Mantei, T., Matthieussent, G., Phys. Fluids 20 (1977) 1005.

[5] Gresillon, D., Mantei, T., Doveil, F., Phys. Rev. Lett. 36 (1976) 1138.

[6] Perrin, J., Schmitt, J. P. M., Chem. Phys. 67 (1982) 167.

[7] Bacal, M., Hamilton, G. W., Phys. Rev. Lett. 42 (1979) 1538.

[8] Bacal, M., Bruneteau, A. M., Graham, W. G., Hamilton, G. W., Nachman, N., J. Appl. Phys. 52 (3) (1981) 1247.
[9] Косн, C., Matthieussent, G., Phys. Fluids 26 (1983) 475.

Koch, C., Matthieussent, G., J. Physique 43 (1982) 67. [10] Leung, K. N., Hershkowitz, N., MaC Kenzie, Phys. Fluids 19 (1976) 1045.

Buzzi, J. M., Snow, J., Hirshfield, J. L., Phys. Lett. A 54 (1975) 344.

Leung, K. N., Samec, T. K., Lam, M., Phys. Lett. A 51 (1975) 490.

[11] Boutry, G. A., Physique Appliquée aux Industries du Vide et de l'Electronique, Tome II (Masson, Paris), 1962.

[12] Mott-Smith, H. M., Langmuir, I., Phys. Rev. 28 (1926) 727.

[13] Gauthereau, C., 1983, Thèse, Orsay, 3562.

[14] Tonks, L., Langmuir, I., Phys. Rev. 34 (1929) 876. 